\title{
Approximation Schemes for two-player pursuit evasion games with visibility constraints
}

\author{
Sourabh Bhattacharya Seth Hutchinson \\ Department of Electrical and Computer Engineering \\ University of Illinois at Urbana Champaign \\ Urbana, Illinois \\ Email: \{sbhattac, seth\}@uiuc.edu
}

\begin{abstract}
In this paper, we consider the problem in which a mobile pursuer attempts to maintain visual contact with an evader as it moves through an environment containing obstacles. This surveillance problem is a variation of traditional pursuitevasion games, with the additional condition that the pursuer immediately loses the game if at any time it loses sight of the evader. We present schemes to approximate the set of initial positions of the pursuer from which it might be able to track the evader.

We first consider the case of an environment containing only polygonal obstacles. We prove that in this case the set of initial pursuer configurations from which it does not lose the game is bounded. Moreover, we provide polynomial time approximation schemes to bound this set. We then extend our results to the case of arbitrary obstacles with smooth boundaries.
\end{abstract}

\section{INTRODUCTION}

Target tracking is an interesting class of motion planning problems. It considers motion strategies for a mobile robot to track a moving target among obstacles. In case of an antagonistic target, the problem lies in the framework of pursuit-evasion which belongs to a special class of problems in game theory. The two players in the game are the pursuer and the evader. The goal of the pursuer is to maintain a line of sight to the evader that is not occluded by any obstacle. The goal of the evader is to escape the visibility region of the pursuer (and break this line of sight) at any instant of time.

This problem has some interesting applications. In security and surveillance systems, tracking strategies enable mobile sensors to monitor moving targets in cluttered environments. In home care settings, a tracking robot can follow elderly people and alert caregivers of emergencies. Target-tracking techniques in the presence of obstacles have been proposed for the graphic animation of digital actors, in order to select the successive viewpoints under which an actor is to be displayed as it moves in its environment [16]. In surgery, controllable cameras could keep a patient's organ or tissue under continuous observation, despite unpredictable motions of potentially obstructing people and instruments.

In this work, we address the problem of a single pursuer trying to maintain visibility of a single evader in a planar environment containing obstacles. The pursuer and the evader have bounded speeds. We address the following question: Given the initial position of the evader, what are the initial positions of the pursuer from which it can track successfully?
We use the term decidable region to refer to the set of initial positions of the pursuer at which the result of the game is known. Similarly, we use the term undecidable region to refer to the set of initial positions of the pursuer at which the result of the game is unknown.

The main contributions of this work are as follows. First; we prove that in an environment containing obstacles, the initial positions of the pursuer from which it can track the evader is bounded. Though this result is trivially true for a bounded workspace, for an unbounded workspace it is intriguing. Second; In this work, we provide polynomial-time approximation schemes to bound the set of initial positions of the pursuer from which it might be able to track successfully. If the initial position of the pursuer lies outside this region, the evader escapes. The size of the region depends on the geometry of the environment and the ratio of the maximum evader speed to the maximum pursuer speed. Third; we address the problem of target tracking in an environment containing non-polygonal obstacles. In the past, researchers [15] have addressed the problem of searching an evader in non-polygonal environments. However, we do not know of any prior work that addresses the problem of tracking an evader in non-polygonal environments. Fourth; although, we do not provide a complete solution to the decidability [5] of the tracking problem in general environments, we present partial solutions by providing polynomial time algorithms to bound the undecidable region.

The rest of the paper is organized as follows. Section II provides the related work. Section III presents the problem formulation. Section IV presents polynomial time approximation schemes to compute the decidable region. Section V extends the approximation schemes to environments containing nonpolygonal obstacles. Section VI presents the conclusions and future research directions.

\section{RELATED WORK}

Some previous work has addressed the motion planning problem for maintaining visibility of a mobile evader. In [4], an algorithm is presented that operates by maximizing the probability of future visibility of the evader. In [14], algorithms are proposed for discrete-time representations of the system in deterministic and stochastic settings. The algorithms become computationally expensive as the number of stages of the 
game is increased. In [8], the authors take into account the positioning uncertainty of the robot pursuer. Game theory is proposed as a framework to formulate the tracking problem, and an approach is proposed that periodically commands the pursuer to move into a region that has no localization uncertainty in order to re-localize and better track the evader afterward.

In [5], the problem of tracking an evader around a single corner is addressed. The free workspace is partitioned according to the strategies used by the players to win the game. The authors have shown that the problem is completely decidable around a single corner. However, in reality, we seldom encounter environments having single corner. Hence the results about a single corner have limited application in real scenarios. In [18], the authors show that the problem of deciding whether or not the pursuer is able to maintain visibility of the evader in a general environment is at least NP-complete. This motivates the necessity to use randomized or approximation techniques to address the problem since any deterministic algorithm would be computationally inefficient.

Some variants of the tracking problem have also been addressed. [7] presents an off-line algorithm that maximizes the evader's minimum time to escape for an evader moving along a known path. In [9][3], a target tracking problem is analyzed for an unpredictable target and an observer lacking prior model of the environment. It computes a risk factor based on the current target position and generates a feedback control law to minimize it. [2] deals with the problem of stealth target tracking where a robot equipped with visual sensors tries to track a moving target among obstacles and, at the same time, remain hidden from the target. Obstacles impede both the tracker's motion and visibility, and also provide hiding places for the tracker. A tracking algorithm is proposed that applies a local greedy strategy and uses only local information from the tracker's visual sensors and assumes no prior knowledge of target tracking motion or a global map of the environment. In [19], the problem of target tracking has been analyzed at a fixed distance between the pursuer and evader. Optimal motion strategies are proposed for a pursuer and evader based on critical events.

Research has been done to track one or more evaders using multiple pursuers. [12] presents a method of tracking several evaders with multiple pursuers in an uncluttered environment. In [11] the problem of tracking multiple targets is addressed using a network of communicating robots and stationary sensors. A region-based approach is introduced which controls robot deployment at two levels, namely, a coarse deployment controller and a target-following controller.

\section{Problem Formulation}

In this paper we consider a mobile pursuer and evader on a plane. They are point robots and move with bounded speeds, $v_{p}(t)$ and $v_{e}(t)$. Therefore, $v_{p}(t):[0, \infty) \rightarrow\left[0, \bar{v}_{p}\right]$ and $v_{e}(t):[0, \infty) \rightarrow\left[0, \bar{v}_{e}\right]$. We use $r$ to denote the ratio of the maximum speed of the evader to that of the pursuer $r=\frac{\bar{v}_{e}}{\bar{v}_{p}}$. The workspace contains obstacles that restrict pursuer and

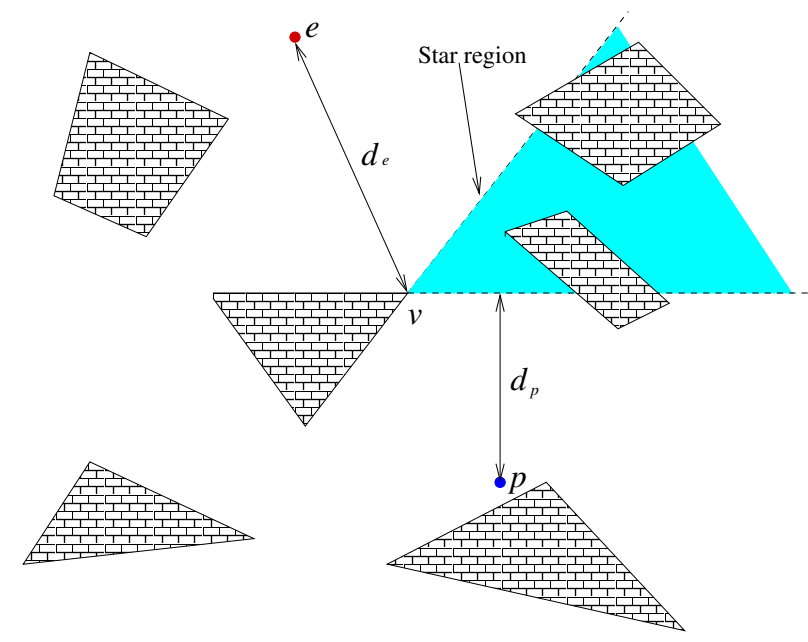

Fig. 1. Star Region associated with the vertex

evader motions and may occlude the pursuer's line of sight to the evader. The initial position of the pursuer and the evader is such that they are visible to each other. To prevent the evader from escaping, the pursuer must keep the evader in its visibility region. The visibility region of the pursuer is the set of points from which a line segment from the pursuer to that point does not intersect the obstacle region. The evader escapes if at any instant of time it can break the line of sight to the pursuer. Visibility extends uniformly in all directions and is only terminated by workspace obstacles (omnidirectional, unbounded visibility).

Now we present a sufficient condition of escape for an evader in general environments. We use it to prove some important results in the next section. The sufficient condition is based on the the concept of a star region. The star region associated with a vertex is defined as the region in the free workspace bounded by the lines supporting the vertex of the obstacle. The shaded region in Figure 1 shows the star region associated with the vertex $v$. The concept of star region is only applicable for a convex vertex (a vertex of angle less than $\pi)$. Using the idea of the star region, a sufficient condition for escape for the evader can be stated as follows.

Sufficient Condition: If the time required by the pursuer to reach the star region associated with a vertex is greater than the time required by the evader to reach the vertex, the evader has a strategy to escape the pursuer's visibility region.

The sufficient condition arises from the fact that if the evader reaches the corner before the pursuer can reach the star region associated with the corner, the evader may escape from the side of the obstacle hidden from the pursuer. This is illustrated in figure 2. In the figure, the evader, $e$, is at the corner while the pursuer, $p$, is yet to reach the star region associated with the corner. If the pursuer approaches the star region from the left side as shown by the solid arrow, the evader can escape the visibility region of the pursuer by 


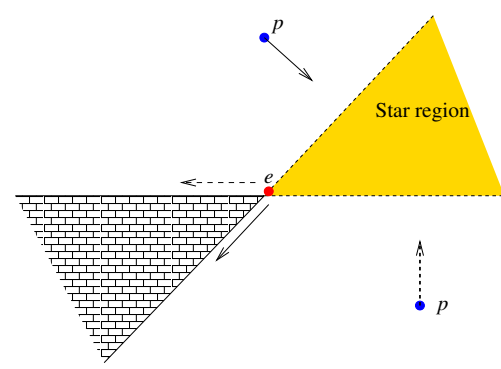

Fig. 2. Sufficient condition for escape

moving in the direction of the solid arrow. On the other hand, if the pursuer approaches the star region from the right side as shown by the dotted arrow, the evader can escape the visibility region of the pursuer by moving in the direction of the dotted arrow.

The relation between the time taken by the pursuer and evader can be expressed in terms of the distances traveled by the pursuer and the evader and their speeds. Referring to Figure 1, if $d_{e}$ is the length of the shortest path of the evader from the corner, $d_{p}$ is the length of the shortest path of the pursuer from the star region associated with the corner and $r$ is the ratio of the maximum speed of the evader to that of the pursuer, the sufficient condition can also be expressed in the following way

SC: If $d_{e}<r d_{p}$, the evader wins the game.

For the sake of convenience, we refer to the sufficient condition as SC in the rest of the paper.

\section{ApProximation SCHEMES For POLYGONAL ENVIRONMENT}

In this section, we show that in any environment containing polygonal obstacles, the set of initial positions from which a pursuer can track the evader is bounded. First, we prove the statement for an environment containing a single convex polygonal obstacle. Then we extend the results to prove in case of a general polygonal environment. This leads to our first approximation scheme. Then we present two more approximation schemes to bound the set of initial positions of the pursuer from which it might be able to track the evader. The results presented in this section hold for unbounded as well as bounded environments.

Consider an evader, $e$, in an environment with a single convex polygonal obstacle having $n$ sides. The edges of the polygonal obstacle are $e_{1}, e_{2} \cdots e_{n}$. Every edge $e_{i}$ is a line segment that lies on a line $l_{e_{i}}$ in the plane. Let $r_{e}=\left(x_{e}, y_{e}\right)$ and $r_{p}=\left(x_{p}, y_{p}\right)$ denote the initial position of the evader and the pursuer respectively. Let $\left\{h_{i}\right\}_{1}^{n}$ denote a family of lines, each given by the equation $h_{i}\left(x, y, r_{e}, r\right)=0$. The presence of the terms $r_{e}$ and $r$ in the equation imply that the equation of the line depends on the initial position of the evader and the speed ratio respectively. Each line $h_{i}$ divides the plane into two half-spaces, namely, $h_{i}^{+}=\left\{(x, y) \mid h_{i}\left(x, y, r_{e}, r\right)>0\right\}$

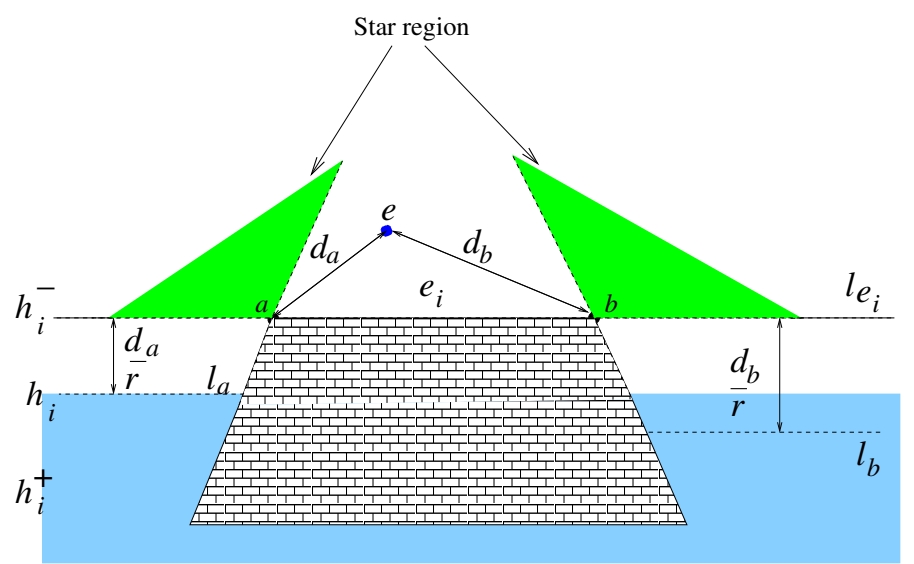

Fig. 3. Proof of Lemma 1

and $h_{i}^{-}=\left\{(x, y) \mid h_{i}\left(x, y, r_{e}, r\right)<0\right\}$. Now we use the SC to prove an important property related to the edges of the obstacle.

Lemma 1: For every edge $e_{i}$, there exists a line $h_{i}$ parallel to $e_{i}$ and a corresponding half-space $h_{i}^{+}$such that the pursuer loses the game if $r_{p} \in h_{i}^{+}$.

Proof: Consider an edge $e_{i}$ of a convex obstacle as shown in Figure 3. Since the obstacle is convex, it lies in one of the half-spaces generated by the line $l_{e_{i}}$. Without the loss of generality, let the obstacle lie in the half-space below the line $l_{e_{i}}$. Let $d_{a}$ and $d_{b}$ be the length of the shortest path of the evader from vertices $a$ and $b$ of the edge $e_{i}$ respectively. Since the obstacle lies in the lower half-space of $l_{e_{i}}$, the star region associated with vertices $a$ and $b$ are in the upper half-space of $l_{e_{i}}$ as shown by the green shaded region. Let $l_{a}$ and $l_{b}$ be the lines at a distance of $\frac{d_{a}}{r}$ and $\frac{d_{b}}{r}$ respectively, from the line $l_{e_{i}}$. If the pursuer lies at a distance $d$ greater than $\min \left(\frac{d_{a}}{r}, \frac{d_{b}}{r}\right)$ below the line $l_{e_{i}}$, then the time taken by the pursuer to reach the line $l_{e_{i}}$ is $t_{p} \geq \frac{d}{\bar{v}_{p}} \geq \frac{\min \left(\frac{d_{a}}{\bar{v}_{p}}, \frac{d_{b}}{r}\right)}{\bar{v}_{p}}$. The minimum time required by the evader to reach corner $a$ or $b$, whichever is nearer, is given by $t_{e}=\frac{\min \left(d_{a}, d_{b}\right)}{\bar{v}_{e}}$. From the expressions of $t_{p}$ and $t_{p}$ we can see that $t_{p}>t_{e}$. Hence the pursuer will reach the nearer of the two corners before the evader reaches line $l_{e_{i}}$. Hence from SC, we conclude that if the pursuer lies below the line $h_{i}$ parallel to $e_{i}$ at a distance of $\min \left(\frac{d_{a}}{r}, \frac{d_{b}}{r}\right)$, then the evader wins the game by following the shortest path to the nearer of the two corners. In Figure 3, since $d_{b}>d_{a}$ the line $h_{i}$ coincides with line $l_{a}$.

Given an edge $e_{i}$ and the initial position of the evader, proof of Lemma 1 provides an algorithm to find the line $h_{i}$ and the corresponding half-plane $h_{i}^{+}$as long as the length of the shortest path of the evader to the corners of an edge is computable. For example, in the presence of other obstacles, the length of the shortest path of the evader to the corners can be obtained by Dijkstra's algorithm.

Now we present some geometrical constructions required to prove the next theorem. Refer to Figure 4. Consider a convex obstacle. Consider a point $c$ strictly inside the obstacle. For 


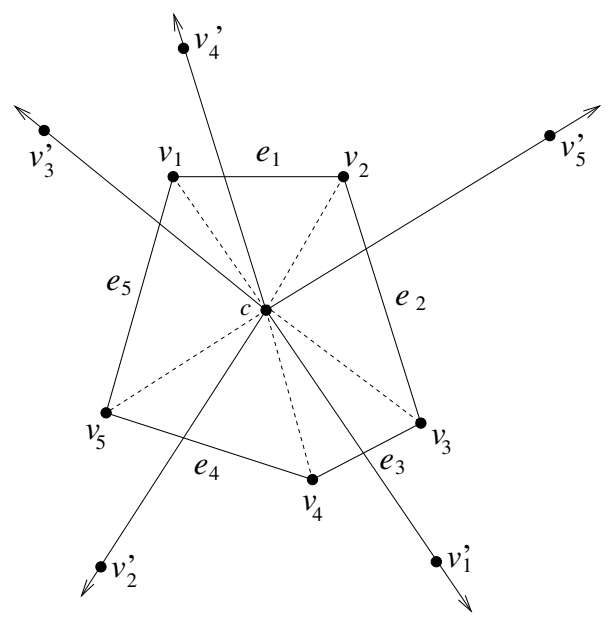

Fig. 4. A polygon and its sectors

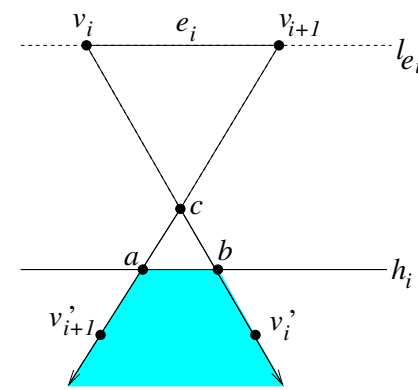

(a)

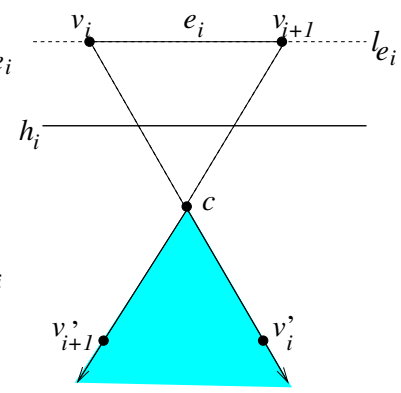

(b)
Fig. 5. Proof of theorem 1

each $i$, extend the line segment $v_{i} c$ to infinity in the direction $\overrightarrow{v_{i}} c$ to form the ray $c v_{i}^{\prime}$. Define the region bounded by rays $c v_{i}^{\prime}$ and $c v_{i+1}^{\prime}$ as sector $v_{i}^{\prime} c v_{i+1}^{\prime}$. The sectors possess the following properties

1) Any two sectors are mutually disjoint.

2) The union of all the sectors is the entire plane.

We can extend the above idea to any $n$ sided convex polygon. We use the construction to prove the following theorem.

Theorem 1: In an environment containing a single convex polygonal obstacle, given the initial position of the evader, the initial positions of the pursuer from which it can win the game is a bounded subset of the free workspace.

Proof: Refer to Figure 5. Consider an edge $e_{i}$ of the convex obstacle with end points $v_{i}$ and $v_{i+1}$. WLOG, the obstacle lies below $l_{e_{i}}$. Let $c$ be a point strictly inside the convex polygon. Extend the line segments $v_{i} c$ and $v_{i+1} c$ to form sector $v_{i}^{\prime} c v_{i+1}^{\prime}$. By Lemma 1, using the initial position of the evader, we can construct a line $h_{i}$ parallel to $e_{i}$ such that if the initial pursuer position lies below $h_{i}$, the evader wins the game. In case the line $h_{i}$ intersects the sector $v_{i}^{\prime} c v_{i+1}^{\prime}$, as shown in Figure 5(a), the evader wins the game if the initial pursuer position lies in the shaded region. In case the line $h_{i}$ does not intersect the sector $v_{i}^{\prime} c v_{i+1}^{\prime}$, as shown in Figure 5(b), the evader wins the game if the initial pursuer position lies anywhere in the sector. Hence for every sector, there is a region of finite area such that if the initial pursuer position lies in it then it might win the game. Every edge of the polygon has a corresponding sector associated with it. Since each sector has a region of finite area such that if the initial pursuer position lies in it, the pursuer might win the game, the union of all these regions is finite. Hence the proposition follows. Figure 6 shows the evader in an environment consisting of a hexagonal obstacle. The polygon in the center bounded by thick lines shows the region of possible pursuer win.

In the proof of theorem 1, we generate a bounded set for each convex polygonal obstacle such that the evader wins the game if the initial position of the pursuer lies outside this set. In a similar way, we can generate a bounded set for a nonconvex obstacle. Given a non-convex obstacle, we construct its convex-hull. We can prove that Lemma 1 holds true for the convex-hull. Finally, we can use Theorem 1 to prove the existence of a bounded set. Due to limitations in space, the proof is omitted.

From the previous discussions, we conclude that any polygonal obstacle, convex or non-convex, restricts the set of initial positions from which the pursuer might win the game, to a bounded set. Moreover, given the initial position of the evader and the ratio of the maximum speed of the evader to the pursuer, the bounded set can be obtained from the geometry of the obstacle by the construction used in the proof of Theorem 1. For any polygon in the environment, let us call the bounded set generated by it, as the $B$ set. If the initial position of the pursuer lies outside the $B$ set, the evader wins the game. For an environment containing multiple polygonal obstacles, we can compute the intersection of all $B$ sets generated by individual obstacles. Since each $B$ set is bounded, the intersection is a bounded set. Moreover, the intersection has the property that if the initial position of the pursuer lies outside the intersection, the evader wins the game. This leads to the following theorem.

Theorem 2: Given the initial position of the evader, the set of initial positions from which the pursuer might win the game is bounded for an environment consisting of polygonal obstacles. Proof: The bounded set referred in this theorem is the intersection of the $B$ sets generated by the obstacles. If the initial pursuer position does not lie in the intersection it implies that it is not contained in all the $B$ sets. Hence there exists at least one polygon in the environment for which the initial pursuer position does not lie in its $B$ set. By Theorem 1, the evader has a winning strategy. Hence the theorem follows.

The intersection of the $B$ sets generated by all the obstacles provides an approximation of the size of the decidable regions. For any initial position of the pursuer outside the intersection, the evader wins the game and hence the result is known. But we still do not know the result of the game for all initial position of the pursuer inside the intersection. However, we can find better approximation schemes and reduce the size of the region in which the result of the game is unknown. In the next subsection, we present one such approximation scheme. 


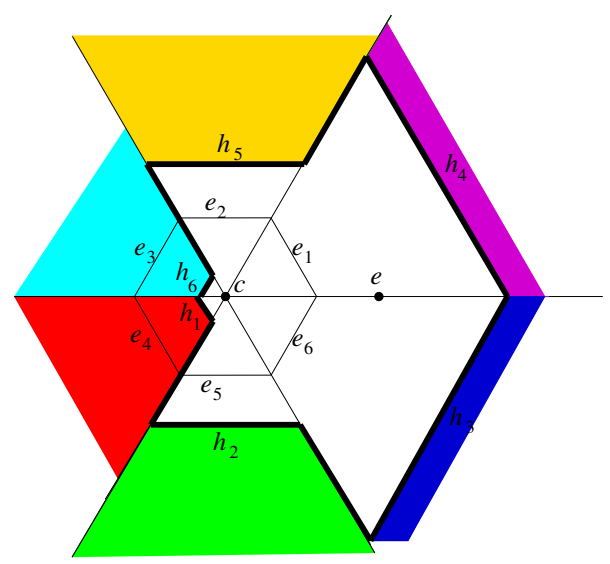

Fig. 6. B set for an environment consisting of a regular hexagonal obstacle and $r=0.5$.

\section{A. U set}

Now we present another approximation scheme that gives a tighter bound of the undecidable region. From Lemma 1, the evader wins the game if $r_{p} \in h_{i}^{+}$for any edge. We can conclude that if $r_{p} \in \cup_{i=1}^{n} h_{i}^{+}$, the evader wins the game. Since $\left(\cup_{i=1}^{n} h_{i}^{+}\right)^{c}=\cap_{i=1}^{n}\left(h_{i}^{+}\right)^{c}=\cap_{i=1}^{n} h_{i}^{-}$, where $S^{c}$ denotes the complement of set $S$, if $r_{p}$ lies outside $\cap_{i=1}^{n} h_{i}^{-}$, the evader wins the game. Hence the set of initial positions from where the pursuer might win the game is contained in $\cap_{i=1}^{n} h_{i}^{-}$. We call $\cap_{i=1}^{n} h_{i}^{-}$as the $U$ set. An important point to note is that the intersection can be taken among any number of halfspaces. If the intersection is among the half-spaces generated by the edges of an obstacle, we call it the $U$ set generated by the obstacle. If the intersection is among the half-spaces generated by all the edges in an environment, we call it the $U$ set generated by the environment.

The next theorem proves that the $U$ set generated by a single obstacle is a subset of the $B$ set and hence a better approximation.

Theorem 3: For a given convex obstacle, the $U$ set is a subset of the $B$ set and hence bounded.

Proof: Consider a point $q$ that does not lie in the $B$ set. From the construction of the $B$ set, $q$ must belong to some half-plane $h_{j}^{+}$. If $q \in h_{j}^{+}$, then $q \notin h_{j}^{-} \Longrightarrow q \notin \cap_{i=1}^{n} h_{i}^{-}$. This implies that the complement of the $B$ set is a subset of the complement of the $U$ set. This implies that the $U$ set is a subset of the $B$ set.

Figure 7 shows the $B$ set and $U$ set for an environment containing a regular hexagonal obstacle. In the appendix, we present a polynomial-time algorithm to compute the $U$ set for an environment with polygonal obstacles. The overall timecomplexity of this algorithm is $O\left(n^{2} \log n\right)$ where $n$ is the number of edges in the environment. Figure 8 shows the evader in a polygonal environment. The region enclosed by the dashed lines is the $U$ set generated by the environment for the initial position of the evader. The $U$ set for any environment having polygonal obstacles is a convex polygon with at most $n$ sides[6]. Figure 9 shows the $U$ set for an environment for

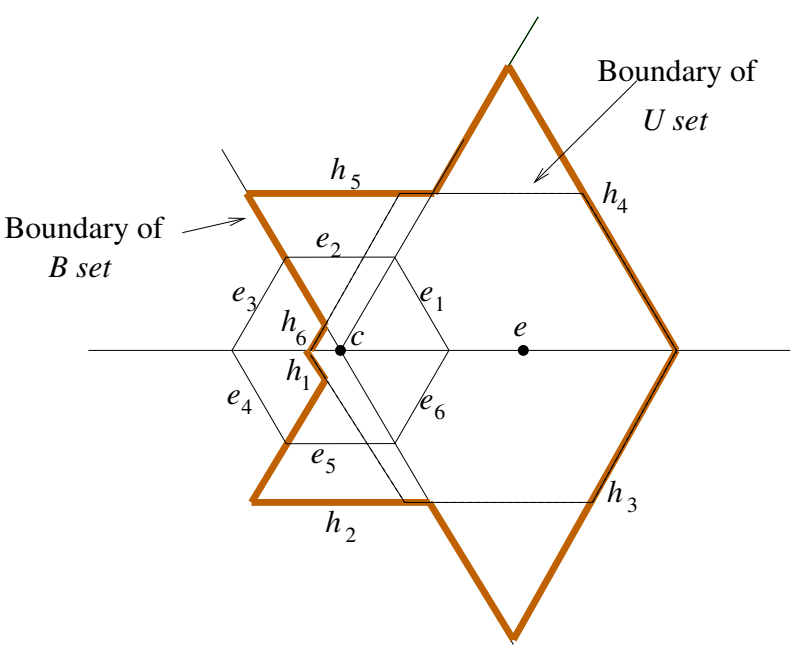

Fig. 7. $B$ set and $U$ set for an environment containing of a regular hexagonal obstacle and $r=0.5$. The polygon bounded by thick lines is the $B$ set and the polygon bounded by thin lines is the $U$ set

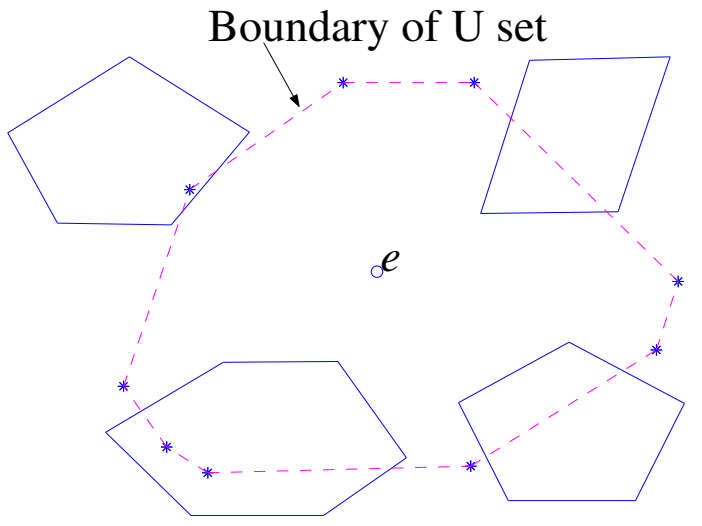

Fig. 8. U set for a general environment

various ratio of the maximum speed of the evader to that of the pursuer. In Figure 9, it can be seen that as the speed ratio between the evader and the pursuer increases, the size of the $U$ set decreases. The size of the $U$ set diminishes to zero at a critical speed ratio. At speed ratios higher than the critical ratio, the evader has a winning strategy for any initial position of the pursuer. Hence the problem becomes decidable [5] when the ratio of the maximum speeds is higher than a critical limit.

The next theorem provides a sufficient condition for escape of the evader in an environment containing obstacles using the $U$ set.

Theorem 4 If the $U$ set does not contain the initial position of either the pursuer or the evader, the evader wins the game. Proof: To prove the theorem we need the following lemma.

Lemma 2: For $r \leq 1$, the evader lies inside the $U$ set.

Proof: For $r \leq 1, \bar{v}_{p} \geq \bar{v}_{e}$. If the pursuer lies at the same position as the evader, its strategy to win is to maintain the same velocity as that of the evader. Hence if the pursuer and the evader have the same initial position, the pursuer can track the evader successfully. Since all the initial positions from 


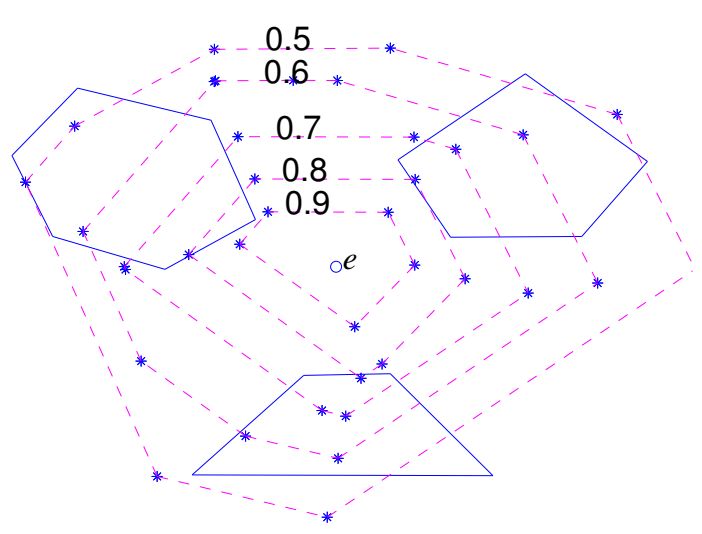

Fig. 9. U set for a various speed ratios of the evader to that of the pursuer

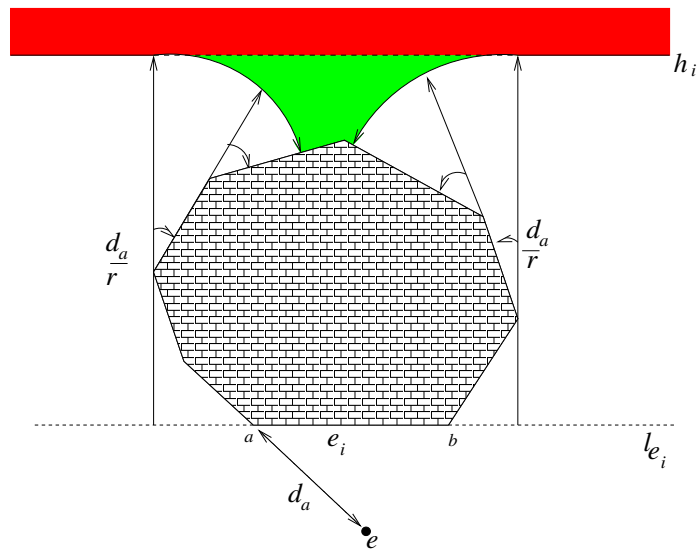

Fig. 10. A polygon in free space. The region shaded in red is obtained by using Lemma 1 . The region shaded in green gets added by using a better approximation scheme.

which the pursuer can win the game must be contained inside the $U$ set, the evader position must also be inside the $U$ set.

Referring back to the proof of Theorem 4, by definition of the $U$ set, if the pursuer lies outside the $U$ set, it loses.

If the evader lies outside the $U$ set, Lemma 2 implies $r>1$. If $r>1, \bar{v}_{e}>\bar{v}_{p}$. If $\bar{v}_{e}>\bar{v}_{p}$, the evader wins the game in any environment containing obstacles. Its winning strategy is to move on the convex hull of any obstacle.

\section{B. Discussion}

In the previous sections, we have provided a simple approximation scheme for computing the set of initial pursuer positions from which the evader can escape based on the intersection of a family of half-spaces. A slight modification to the proposed scheme leads to a better approximation. In the proof of Lemma 1, we presented an algorithm to find a half-space for every edge of the polygon such that if the initial position of the pursuer lies in the half-space, the evader wins the game. All the points in the half-space are at a distance greater than $\frac{d_{a}}{r}$ from $l_{e_{i}}$. By imposing the condition that the minimum distance of the desired set of points from $l_{e_{i}}$ in the free workspace should be greater than $\frac{d_{a}}{r}$, we can include

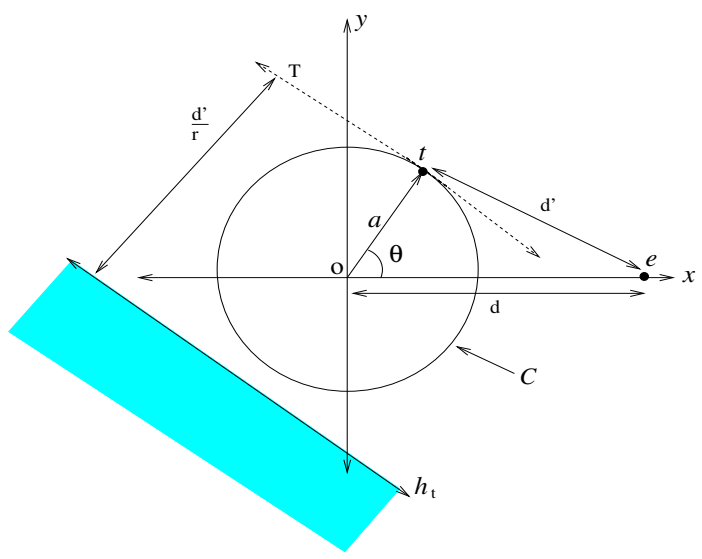

Fig. 11. A circular obstacle in free space

more points in the decidable regions as shown in Figure 10. The figure shows an obstacle in free space. From the proof of Lemma 1, we get the half-space shaded in red. By adding the new condition, the region shaded in green gets included. When we repeat this for every edge, the set of initial positions from which the pursuer might win the game gets reduced and leads to a better approximation of the decidable regions. The boundary of the shaded region consists of straight lines and arc of circles. The boundary of the desired set is obtained by computing the intersections among a bunch of rays and arcs of circles generated by each edge. In this case a better approximation comes at the cost of expensive computation. We believe that better approximation schemes exist and one of our ongoing efforts is in the direction of obtaining computationally efficient approximation schemes.

None of the approximation schemes we have suggested so far restrict the initial position of the pursuer to be in the evader's visibility region. This condition can be imposed by taking an intersection of the output of the approximation algorithm with the visibility polygon at the evader's initial position. Efficient algorithms exist for computing the visibility polygon of a static point in an environment[10].

In the next section we extend the idea of $U$ set to environments containing non-polygonal obstacles.

\section{APPROXIMATION METHODS FOR NON-POLYGONAL OBSTACLES}

In this section we extend the approximation schemes presented in the previous section to non-polygonal obstacles. In order to illustrate the techniques required to handle nonpolygonal obstacles, we compute an approximation for the initial positions of the pursuer from which the evader wins the game for the simple case of an evader in an environment containing a circular obstacle. Then we present the algorithm for any environment containing convex obstacles with smooth boundaries.

Figure 11 shows an evader, $e$, in an environment containing a circular obstacle of radius $a$ in free space. The boundary of the obstacle is denoted by $C$. Let $t$ be a point on $C$ such that $\angle O t e=\theta$ and $|t e|=d^{\prime} . T$ denotes the tangent to $C$ at $t$. Let 
$h_{t}$ be a line at a distance of $\frac{d^{\prime}}{r}$ from $T$ on the same side of $T$ as the obstacle. By Lemma 1, the evader wins the game if the pursuer lies in the half-space $h_{t}^{+}$, shown by the shaded region. The equation of line $h_{t}$ is $y+x \cot \theta-\left(a-\frac{d^{\prime}}{r}\right) \csc \theta=0$. For every point $t$ on $C$, there exists a line $h_{t}$ and the corresponding half-space $h_{t}^{+}$such that if the initial position of the pursuer lies in $h_{t}^{+}$, the evader wins the game. Hence if the initial pursuer position lies in $\cup_{t \in C} h_{t}^{+}$, the evader wins the game $\Longrightarrow$ if the initial pursuer position lies outside $\cap_{t \in C} h_{t}^{-}$, the evader wins the game. Let us call $\cap_{t \in C} h_{t}^{-}$as the $U$ set.

Now we compute the boundary of the $U$ set. Let $l(x, y, \theta)$ denote the family of lines $h_{t}$ generated by all points $t$ lying on $C$. Due to symmetry of the environment about the $x$ axis, the $U$ set is symmetric about the $x$-axis. We present the construction of the boundary of the $U$ set generated as $\theta$ increases from 0 to $\pi$. Let $\partial U$ denote the boundary of the $U$ set.

Theorem 6- $\partial U$ is the envelope of the family of lines $l(x, y, \theta)$.

Proof: Consider any point $q$ on $\partial U$. Since $q$ belongs to the boundary of the $U$ set, it belongs to some line, $h_{q}$, in the family $l(x, y, \theta)$. Either $h_{q}$ is tangent to $\partial U$ or else it intersects $\partial U$. In case it intersects $\partial U$, there is a neighborhood around $q$ in which $\partial U$ lies in both the half-spaces generated by $h_{q}$. This is not possible since one of the half-spaces generated by $h_{q}$ has to be entirely outside the $U$ set. Hence $h_{q}$ is tangent to $\partial U$. Since $q$ is any point on $B$, it implies that for all points $q$ on $\partial U$, the tangent to $\partial U$ at $q$ belongs to the family $l(x, y, \theta)$. A curve satisfying this property is the envelope to the family of lines $l(x, y, \theta)$. Hence the proposition follows.

Using the Envelope theorems [20], the envelope of a family of lines $l(x, y, \theta)$ can be obtained by solving the following equations simultaneously

$$
\begin{aligned}
l(x, y, \theta)=y+x \cot \theta-\left(a-\frac{d^{\prime}}{r}\right) \csc \theta & =0 \\
\frac{\partial l}{\partial \theta} & =0
\end{aligned}
$$

$d^{\prime}$ as a function of $\theta$ is given by

$$
d^{\prime}(\theta)=\left\{\begin{array}{lll}
\sqrt{a^{2}+d^{2}-2 a d \cos \theta} & \text { if } \quad \theta \leq \theta_{0} \\
\sqrt{d^{2}-a^{2}}+a\left(\theta-\theta_{0}\right) & \text { if } \quad \theta \geq \theta_{0}
\end{array}\right.
$$

where $\theta_{0}=\cos ^{-1} \frac{a}{d}$.

The solution is

A. Case $1\left(\theta \leq \theta_{0}\right)$

$$
\begin{aligned}
& x=\left(a-\frac{\sqrt{a^{2}+d^{2}-2 a d \cos \theta}}{r}\right) \cos \theta+\frac{a d \sin ^{2} \theta}{r \sqrt{a^{2}+d^{2}-2 a d \cos \theta}} \\
& y=\left(a-\frac{\sqrt{a^{2}+d^{2}-2 a d \cos \theta}}{r}\right) \sin \theta-\frac{a d \sin \theta \cos \theta}{r \sqrt{a^{2}+d^{2}-2 a d \cos \theta}}
\end{aligned}
$$

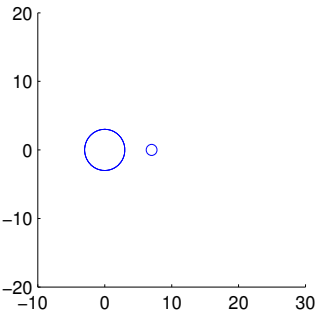

(a)

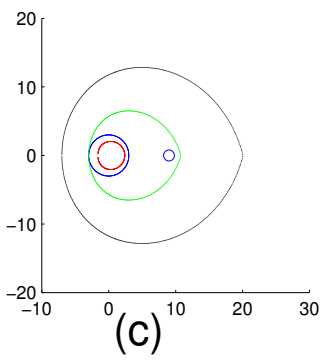

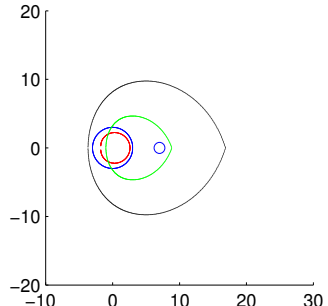

(b)

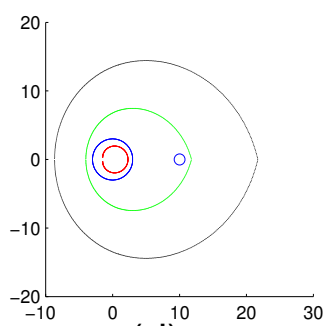

(d)
Fig. 12. (a) shows a circular obstacle with the initial position of the evader. The smaller circle is the evader. In (b),(c) and (d), $d=5,7$ and 9 units respectively. In each of the figures (b), (c) and (d), the black boundary is for $r=0.5$, the green boundary is $r=1$ and the red boundary is for $r=10$

\section{B. Case $2\left(\pi \geq \theta \geq \theta_{0}\right)$}

$$
\begin{aligned}
& x=\left(a-\frac{\sqrt{d^{2}-a^{2}}+a\left(\theta-\theta_{0}\right)}{r}\right) \cos \theta+\frac{\sin \theta}{r} \\
& y=\left(a-\frac{\sqrt{d^{2}-a^{2}}+a\left(\theta-\theta_{0}\right)}{r}\right) \sin \theta-\frac{\cos \theta}{r}
\end{aligned}
$$

Since $\partial U$ is symmetrical about the $x$-axis, the other half of $\partial U$ is obtained by reflecting the above curves about the $x$-axis. Figure 12(a) shows an evader in an environment consisting of a disc-like obstacle. Figures 12(b),(c) and (d) show the boundary of the $U$ set for varying distance between the evader and the obstacle. In each of these figures, the boundary of the $U$ set is shown for three different values of $r$. We can see that for $r \leq 1$, the evader lies inside the $U$ set as given by Lemma 2 .

The above procedure can be used to construct the $U$ set for any convex obstacle with smooth boundary. Given the initial position of the evader, we present the procedure to construct the boundary of the $U$ set for a obstacle with smooth boundary.

Consider an obstacle with smooth boundary given by the equation $f(x, y)=0$. The procedure to generate the boundary of the $U$ set is as follows

1) Given any point $t$ on the boundary, compute the minimum distance of the point from the evader. Let it be $d_{t}$.

2) Find the equation of the line $h_{t}$ at a distance of $\frac{d_{t}}{r}$ from the tangent to the obstacle at $t$.

3 ) Find the family $l(x, y, \theta)$ of lines generated by $h_{t}$ as $t$ varies along the boundary of the obstacle. $\theta$ is a parameter that defines $t$. 
4) Compute the envelope of the family $l(x, y, \theta)$. This is the boundary of the $U$ set. This is true since the proof of Theorem 5 does not depend on the shape of the obstacle.

\section{CONCLUSION AND Future RESEARCH}

In this work we address the problem of target-tracking in general environments. We prove that in a general environment containing obstacles, given the initial position of the evader, the set of initial positions from which the pursuer might be able to track the evader is bounded. Moreover we provide an approximation algorithm to construct a convex polygonal region to bound that region. We provide a sufficient condition for escape of the evader in a general polygonal environment that depends on the geometry of the obstacles, the initial position of the evader and the ratio of the maximum speed of the evader to that of the pursuer. We extend the approximation schemes to obstacles with smooth boundaries.

Given the complete map of the environment, our results depend only on the initial position and the maximum speeds of the pursuer and evader. Hence our results hold for various settings of the problem such as an unpredictable or predictable evader [14] or localization uncertainties in the future positions of the players [8] or delay in pursuer's sensing abilities [17].

In the future, we would like to provide an algorithm to approximate the initial positions of the pursuer from which it can track the evader and also the strategies used by the pursuer to track successfully. We are using game-theory as a framework to provide feedback strategies for the pursuer to track successfully. We are also investigating the problem of target-tracking with multiple pursuers.

An interesting direction of future research would be to extend our results to the target-tracking in $\mathbb{R}^{3}$. Researchers have addressed the problem of target-tracking in $\mathbb{R}^{3}$ [1]. We believe that some of our results can be used in 3-d by considering polyhedrons as bounding sets instead of polygons. Another direction of future research would be to incorporate dynamics in the player's motion model.

\section{REFERENCES}

[1] T. Bandyopadhyay, M.H. Ang Jr, and D. Hsu. Motion planning for 3-D target tracking among obstacles. International Symposium on Robotics Research, 2007.

[2] T. Bandyopadhyay, Y. Li, M.H. Ang Jr, and D. Hsu. Stealth Tracking of an Unpredictable Target among Obstacles. Proceedings of the International Workshop on the Algorithmic Foundations of Robotics, 2004.

[3] T. Bandyopadhyay, Y. Li, M.H. Ang Jr., and D Hsu. A Greedy Strategy for Tracking a locally Predicatable Target among Obstacles. Robotics and Automation, Proceedings. ICRA'O2. IEEE International Conference on, pages 2342-2347, 2006.

[4] C. Becker, H. Gonzalez-Banos, J.C. Latombe, and C. Tomasi. An intelligent observer. Proceedings of International Symposium on Experimental Robotics, pages 94-99, 1995.

[5] Sourabh Bhattacharya, Salvatore Candido, and Seth Hutchinson. Motion strategies for surviellance. In Robotics: Science and Systems - III, 2007.

[6] M. de Berg, M. van Kreveld, M. Overmars, and O. Schwarzkopf. Computational Geometry- Algorithms and Applicaions. Springer-Verlag, Berlin Heidelberg, 1997.

[7] A. Efrat, HH Gonzalez-Banos, SG Kobourov, and L. Palaniappan. Optimal strategies to track and capture a predictable target. Robotics and Automation, 2003. Proceedings. ICRA'O3. IEEE International Conference on, 3, 2003.
[8] P. Fabiani and J.C. Latombe. Tracking a partially predictable object with uncertainty and visibility constraints: a game-theoretic approach. Technical report, Technical report, Univeristy of Stanford, December 1998. http://underdog. stanford. edu/.(cited on page 76).

[9] HH Gonzalez-Banos, C.Y. Lee, and J.C. Latombe. Real-time combinatorial tracking of a target moving unpredictably among obstacles. Robotics and Automation, 2002. Proceedings. ICRA'02. IEEE International Conference on, 2, 2002.

[10] J. E. Goodman and J. O. Rourke. Handbook of Discrete and Computational Geometry. CRC Press, New York, 1997.

[11] B. Jung and G.S. Sukhatme. Tracking Targets Using Multiple Robots: The Effect of Environment Occlusion. Autonomous Robots, 13(3):191205, 2002.

[12] Parker L. Algorithms for Multi-Robot Observation of Multiple Targets. Journal on Autonomous Robots, 12:231-255, 2002.

[13] J. P. Laumond, S. Sekhavat, and F. Lamiraux. Guidelines in Nonholonomic motion planning for Mobile Robots. Springer, 1998.

[14] S. M. LaValle, H. H. Gonzalez-Banos, C. Becker, and J. C. Latombe. Motion strategies for maintaining visibility of a moving target. In Robotics and Automation, 1997. Proceedings., 1997 IEEE International Conference on, volume 1, pages 731-736, Albuquerque, NM, USA, April 1997.

[15] S. M. LaValle and J. Hinrichsen. Visibility-based pursuit-evasion: The case of curved environments. IEEE Transactions on Robotics and Automation, 17(2):196-201, April 2001.

[16] T.Y. Li, J.M. Lien, S.Y. Chiu, and T.H. Yu. Automatically generating virtual guided tours. Computer Animation Conference, pages 99-106, 1997.

[17] R. Murrieta, A. Sarmiento, and S. Hutchinson. On the existence of a strategy to maintain a moving target within the sensing range of an observer reacting with delay. Intelligent Robots and Systems, 2003.(IROS 2003). Proceedings. 2003 IEEE/RSJ International Conference on, 2, 2003.

[18] R. Murrieta-Cid, R. Monroy, S. Hutchinson, and J. P. Laumond. A complexity result for the pursuit-evasion game of maintaining visibility of a moving evader. Accepted in IEEE International Conference on Robotics and Automation, 2008.

[19] R. Murrieta-Cid, T. Muppirala, A. Sarmiento, S. Bhattacharya, and S. Hutchinson. Surveillance strategies for a pursuer with finite sensor range. International Journal of Robotics Research, pages 1548-1553, 2007.

[20] E. Silberberg. The Viner-Wong Envelope Theorem. Journal of Economic Education, 30(1):75-79, 1999

\section{APPENDIX}

\section{A. Algorithm for generating the U-set}

Algorithm CONSTRUCTUSET(S, $r,\left(x_{e}, y_{e}\right)$ )

Input: $\mathrm{A}$ set $\mathrm{S}$ of disjoint polygonal obstacles, the evader position $r_{e}=\left(x_{e}, y_{e}\right)$, ratio of maximum evader speed to maximum pursuer speed $r$

Output: The coordinates of the vertices of the $U$ set

1) For every edge $e_{i}$ in the environment with end-points $a_{i}, b_{i}$

2) $\quad l_{1}=\operatorname{DIJKSTRA}\left(\mathrm{VG}(\mathrm{S}), r_{e}, a_{i}\right)$

3) $\quad l_{2}=\operatorname{DIJKSTRA}\left(\mathrm{VG}(\mathrm{S}), r_{e}, b_{i}\right)$

4) $\quad d_{e_{i}}=\frac{\min \left(l_{1}, l_{2}\right)}{r}$

5) Find the equation of $h_{i}$ using Lemma 1 .

6) INTERSECTHALFPLANES $\left(h_{1}^{-}, \ldots . h_{n}^{-}\right)$

The subroutine $\mathrm{VG}(\mathrm{S})$, computes the visibility graph of the environment S. The subroutine $\operatorname{DIJKSTRA}(\mathrm{G}, \mathrm{I}, \mathrm{F})$ computes the least distance between nodes $I$ and $F$ in graph $G$. The subroutine INTERSECTHALFPLANES $\left(h_{1}^{-}, \ldots, h_{n}^{-}\right)$computes the intersection of the half planes $h_{1}^{-}, \ldots, h_{n}^{-}$[6]. The time complexity of the above algorithm is $O\left(n^{2} \log n\right)$, where $n$ is the number of edges in the environment. 